\title{
Strict error bounds for linear and nonlinear solid mechanics problems using a patch-based flux-free method
}

\author{
Régis Cottereau ${ }^{1, a}$, Ludovic Chamoin ${ }^{2}$ and Pedro Díez ${ }^{3}$ \\ 1 Laboratoire MSSMat, École Centrale Paris, CNRS, Grande voie des vignes, 92295 Châtenay-Malabry, France \\ 2 LMT-Cachan, ENS Cachan, CNRS, Paris 6 University, 61 avenue du Président Wilson, 94230 Cachan, France \\ 3 Laboratori de Càlcul Numèric, Universitat Politècnica de Catalunya, Jordi Girona 1-3, 08034 Barcelona, Spain
}

\begin{abstract}
We discuss, in this paper, a common flux-free method for the computation of strict error bounds for linear and nonlinear finite-element computations. In the linear case, the error bounds are on the energy norm of the error, while, in the nonlinear case, the concept of error in constitutive relation is used. In both cases, the error bounds are strict in the sense that they refer to the exact solution of the continuous equations, rather than to some FE computation over a refined mesh. For both linear and nonlinear solid mechanics, this method is based on the computation of a statically admissible stress field, which is performed as a series of local problems on patches of elements. There is no requirement to solve a previous problem of flux equilibration globally, as happens with other methods.
\end{abstract}

Key words: Verification / a posteriori error estimation / error bounds / residual-based estimators / fluxfree error estimator

\section{Introduction}

In the past few decades, research and industry in the field of mechanics have relied increasingly on computational tools. The models and the resolution methods have grown increasingly complex and their careful assessment has become unavoidable. In particular, the error arising from the resolution of equations defined on a continuum by the finite-element (FE) method has to be estimated and controlled (the so-called "verification"). Hence, this paper describes a technique for the estimation of bounds on the energy norm of that error, in the particular setting of linear and nonlinear solid mechanics.

This technique is an a posteriori error estimation method, which means that it uses the output of the FE computation to assess its accuracy. Three groups of techniques exist within that general class (see [1] for a more detailed review, and [2] for a recent journal special issue on the subject): one based on the so-called constitutive relation error, by Ladevèze and co-workers (see for example [3-6]); another based on the comparison of the discontinuous stress field computed by the FE method and a regularized version, following the leading work of Zienkiewicz and Zhu [7]; and, finally, a large family of methods, generically called implicit residual methods, which are based on the

\footnotetext{
${ }^{a}$ Corresponding author: pedro.diez@upc.edu
}

(approximate) resolution of a residual error equation on a series of small local problems with appropriate boundary conditions (see for example [8,9], and comparisons between approaches in [10-12]). Among these methods, we distinguish between the hybrid-flux methods (also called equilibrated residual methods), where the local problems are element-based, and the flux-free techniques [8,12-17], where the subdomains are patches of elements. The advantage of the latter is that the boundary conditions on the local problems are trivial, and that they do not require any flux equilibration. Similarly, the constitutive relation error method requires the computation of a statisticallyadmissible stress field, that can be constructed either using some hybrid-flux [3] or flux-free method [6].

In the case of implicit residual methods, the error is defined as the energy norm of the difference between the exact and approximate fields. In most of the subdomainbased methods, although the local error estimation problems are posed on smaller geometrical spaces, the functional spaces involved are still infinite-dimensional. The exact error is therefore usually estimated as the solution of an alternate FE problem, posed on a much larger space than the original FE computation. Hence, the bounds computed are given with respect to a "refined" solution and are valid only asymptotically. However, it is much more interesting, from an engineering point of view, to provide strict bounds, that is to say 
with respect to the exact error. A dual formulation was proposed to attain that goal in the context of hybridflux residual estimators $[18,19]$, and was later extended to the flux-free error estimators in the case of transient convection-reaction-diffusion problems [20,21] and linear solid mechanics [22]. To this day, however, no such strict bounds have been encountered in the case of nonlinear mechanics.

On the contrary, using the constitutive relation error approach, it is possible to provide strict bounds for nonlinear elasticity. In particular, using the concept of dissipation error, it is possible to obtain bounds related to the conjugate dissipation pseudopotential of the difference between the exact stress field and the mean of the FE stress field and a statically admissible field [4,5]. We propose in this paper to compute these bounds using the flux-free method developed in [22] for the computation of the statically admissible field. This yields a common framework for the computation of the statically admissible field used for the derivation of strict bounds in both linear and nonlinear solid mechanics. The objectives of this paper can be seen in two equivalent ways: (1) the extension of the method proposed in [22] to the nonlinear realm, or (2) a new method, within the context of the constitutive relation error, to compute the admissible stress field used to derive strict bounds on the error.

The paper starts with a summary of the main ideas in [22]. In particular, the local dual problems yielding the global statically admissible stress field are presented. In the following section, the concept of dissipation error is introduced, and the use of the stress field computed in the previous section to derive strict bounds on the error is presented. Finally two applications are developed, that show the interest of the method, and in particular the accuracy of the bounds obtained.

\section{Strict flux-free error estimator for linear elasticity}

We consider a general problem of elasticity, posed on a domain $\Omega$ of $\mathbb{R}^{\mathrm{d}}$, and written, in strong form: find $\boldsymbol{u}_{\mathrm{ex}}$ : $\Omega \rightarrow \mathbb{R}^{\mathrm{d}}$, such that

$$
\begin{cases}\operatorname{Div}_{\boldsymbol{x}} \boldsymbol{\sigma}\left(\boldsymbol{u}_{\mathrm{ex}}\right)+\boldsymbol{f}=\mathbf{0} & \text { in } \Omega \\ \boldsymbol{\sigma}\left(\boldsymbol{u}_{\mathrm{ex}}\right) \cdot \boldsymbol{n}=\boldsymbol{g} & \text { on } \Gamma^{\mathrm{N}} \\ \boldsymbol{u}_{\mathrm{ex}}=\mathbf{0} & \text { on } \Gamma^{\mathrm{D}}\end{cases}
$$

where $\boldsymbol{\sigma}(\boldsymbol{w})=\mathcal{C}(\boldsymbol{\epsilon}(\boldsymbol{w}))$ is the Cauchy stress tensor associated to the displacement field $\boldsymbol{w}, \boldsymbol{f}$ and $\boldsymbol{g}$ are load fields, respectively over the volume and the boundary, and $\Gamma^{\mathrm{D}}$ and $\Gamma^{\mathrm{N}}$ are parts of the boundary $\partial \Omega$ of $\Omega$ on which boundary conditions are imposed, respectively Dirichlet et Neumann, and such that $\partial \Omega=\overline{\Gamma^{\mathrm{D}} \cup \Gamma^{\mathrm{N}}}$ and $\Gamma^{\mathrm{D}} \cap \Gamma^{\mathrm{N}}=\emptyset$. Throughout the paper, the small strains assumption will be made. In this section of the paper, the fourth-order tensor enforcing the constitutive relation $\mathcal{C}$ is assumed linear, and denoted $\mathcal{K}$, with the usual hypotheses of symmetry $\left(\mathcal{K}_{i j k l}=\mathcal{K}_{k l i j}=\mathcal{K}_{j i k l}\right)$ and positivedefiniteness $\left(\mathcal{K}_{i j k l} \epsilon_{i j} \epsilon_{k l}>\alpha \epsilon_{i j} \epsilon_{i j}, \alpha>0\right.$, for any secondorder real symmetric tensor $\epsilon$ ). In the next section, a visco-elastic constitutive relation will be considered.
As the solution $\boldsymbol{u}_{\text {ex }}$ is usually not available, an approximation can be sought using the FE method. This method is based on a weak form of the previous equations, using a smaller functional space for the solutions: find $\boldsymbol{u}_{\mathrm{H}} \in \mathcal{V}_{\mathrm{H}}$, such that

$$
a_{\Omega}\left(\boldsymbol{u}_{\mathrm{H}}, \boldsymbol{v}\right)=\ell(\boldsymbol{v}), \forall \boldsymbol{v} \in \mathcal{V}_{\mathrm{H}}
$$

where $a_{\Omega}:\left[\mathcal{H}^{1}(\Omega)\right]^{\mathrm{d}} \times\left[\mathcal{H}^{1}(\Omega)\right]^{\mathrm{d}} \rightarrow \mathbb{R}$ is given by $a_{\Omega}(\boldsymbol{w}, \boldsymbol{v})=\int_{\Omega} \boldsymbol{\sigma}(\boldsymbol{w}): \boldsymbol{\epsilon}(\boldsymbol{v}) \mathrm{d} \Omega$ and $\ell:\left[\mathcal{H}^{1}(\Omega)\right]^{\mathrm{d}} \rightarrow \mathbb{R}$ is given by $\ell(\boldsymbol{v})=\int_{\Omega} \boldsymbol{f} \cdot \boldsymbol{v} \mathrm{d} \Omega+\int_{\Gamma^{\mathrm{N}}} \boldsymbol{g} \cdot \boldsymbol{v} \mathrm{d} \Gamma$. In practice, $\mathcal{V}_{\mathrm{H}}$ is often chosen as the functional space composed of functions that are linear over each element of a mesh. For reasons that will be described further down, it is necessary here to use functions that are at least quadratic over the elements.

Once that approximate solution has been computed, the objective of a posteriori error estimation method is to provide indications on the accuracy of that approximation. The best possible indicator should give bounds that are at the same time conservative, with respect to the exact solution of the problem (1), accurate, and cheap to compute. The method that we describe in the first part of this paper allows to obtain such bounds on the energetic norm of the error $\left\|\boldsymbol{u}_{\mathrm{H}}-\boldsymbol{u}_{\mathrm{ex}}\right\|_{\Omega}^{2}=\|\boldsymbol{e}\|_{\Omega}^{2}=a_{\Omega}(\boldsymbol{e}, \boldsymbol{e})$. Similar bounds can then classically be obtained for a large class of quantities of interest $[23,24]$.

Following the principles described in $[12,19,22]$, the bounds are obtained through the resolution of local problems posed on subdomains $\Omega_{i}$ of $\Omega$. These subdomains $\Omega_{i}$ are called patches or stars, and defined as the set of elements touching the node $i$ of the original mesh. The local problems to be solved are: find, for each vertex $i$ of the original mesh, a stress tensor $\boldsymbol{q}^{i}$, such that

$$
\begin{cases}\operatorname{Div}_{\boldsymbol{x}} \boldsymbol{q}^{i}+\phi_{i}\left(\boldsymbol{f}+\operatorname{Div}_{\boldsymbol{x}} \boldsymbol{\sigma}\left(\boldsymbol{u}_{\mathrm{H}}\right)\right)=\mathbf{0} & \text { in } \Omega_{i} \\ \llbracket \boldsymbol{q}^{i} \cdot \boldsymbol{n} \rrbracket=-\phi_{i} \llbracket \boldsymbol{\sigma}\left(\boldsymbol{u}_{\mathrm{H}}\right) \cdot \boldsymbol{n} \rrbracket & \text { on } \Gamma_{i} \backslash \partial \Omega_{i} \\ \boldsymbol{q}^{i} \cdot \boldsymbol{n}=\phi_{i}\left(\boldsymbol{g}-\boldsymbol{\sigma}\left(\boldsymbol{u}_{\mathrm{H}}\right) \cdot \boldsymbol{n}\right) & \text { on } \Gamma^{\mathrm{N}} \cap \partial \Omega_{i} \\ \boldsymbol{q}^{i} \cdot \boldsymbol{n}=\mathbf{0} & \text { on } \partial \Omega_{i} \backslash \Gamma^{\mathrm{N}}\end{cases}
$$

where $\phi_{i}$ is the linear FE interpolation function associated to the node $i$ of the mesh, and $\Gamma_{i}$ is the union of the boundaries of the elements forming the star $\Omega_{i}$ (hence $\Gamma_{i} \backslash \partial \Omega_{i}$ represents the internal boundaries of the star $\Omega_{i}$ ). The main point of these local problems is that they are defined automatically from the original problem. In particular, the definition of the loading terms $\phi_{i}\left(\boldsymbol{f}+\operatorname{Div}_{\boldsymbol{x}} \boldsymbol{\sigma}\left(\boldsymbol{u}_{\mathrm{H}}\right)\right)$, $-\phi_{i} \llbracket \boldsymbol{\sigma}\left(\boldsymbol{u}_{\mathrm{H}}\right) \cdot \boldsymbol{n} \rrbracket$ and $\phi_{i}\left(\boldsymbol{g}-\boldsymbol{\sigma}\left(\boldsymbol{u}_{\mathrm{H}}\right) \cdot \boldsymbol{n}\right)$ does not require the resolution of an intermediate flux equilibration problem, as with other methods. However, to ensure the existence of a solution to these problems, it is necessary that the original problem be solved in a quadratic space of function over each element. Basically, the idea of the proof (developed fully in [22]) is that both forces and moments arising from the volume and surface loads in problems (2) should be equilibrated. This imposes that the function $\boldsymbol{\sigma}\left(\boldsymbol{u}_{\mathrm{H}}\right)$ be linear over each element, and hence that $\boldsymbol{u}_{\mathrm{H}}$ be quadratic. It should also be noted that, to obtain the desired bound, it is necessary to impose on the boundary $\partial \Omega_{i} \backslash \Gamma^{\mathrm{N}}$, which intersects the Dirichlet boundary of 
the original problem, a homogeneous Neumann boundary condition. This may slightly deteriorate the local efficiency index (ratio of the estimated error on the exact error, which are the two quantities described in Eq. (3), below) in some cases, but only slightly and in the vicinity of that boundary (see the dam example in [22]).

Once the local problems (2) have been solved, it can be shown that (see [22] for a proof)

$$
\|\boldsymbol{e}\|_{\Omega}^{2} \leq 2 \pi_{\mathrm{c}}\left(\sum_{i} \boldsymbol{q}^{i}\right)
$$

where $\pi_{\mathrm{c}}(\boldsymbol{q})=\int_{\Omega} \boldsymbol{q}: \mathcal{C}^{-1}(\boldsymbol{q}) \mathrm{d} \Omega$ is the complementary energy associated to the stress tensor $\boldsymbol{q}$. It can also be shown that the solutions $\boldsymbol{q}^{i}$ of the local problems are not defined in a unique manner, so that different (local) strategies can be proposed to (globally) improve the error bound. Equation (3) is the reason why we call the obtained bound "strict", in the sense that the reference is indeed the exact error $\|\boldsymbol{e}\|_{\Omega}=\left\|\boldsymbol{u}_{\mathrm{ex}}-\boldsymbol{u}_{\mathrm{H}}\right\|_{\Omega}$, and not some error $\left\|\boldsymbol{u}_{\mathrm{h}}-\boldsymbol{u}_{\mathrm{H}}\right\|_{\Omega}$, with respect to a solution $\boldsymbol{u}_{h}$ obtained over a refined mesh.

\section{Strict flux-free error estimator for nonlinear elasticity}

The proof of the bounding Equation (3) is based among other things on an hypothesis of linearity on the error $\boldsymbol{\sigma}(\boldsymbol{e})=\boldsymbol{\sigma}\left(\boldsymbol{u}_{\mathrm{ex}}-\boldsymbol{u}_{\mathrm{H}}\right)=\boldsymbol{\sigma}\left(\boldsymbol{u}_{\mathrm{ex}}\right)-\boldsymbol{\sigma}\left(\boldsymbol{u}_{\mathrm{H}}\right)$, which is not anymore true for nonlinear elasticity problems. Rather than the classical framework of the error in solution considered in the previous section, we now follow the theoretical framework of the error in constitutive relation [3-5].

The basic principle in this approach consists in finding a displacement-stress pair $\left(\boldsymbol{u}_{\mathrm{H}}, \boldsymbol{q}^{*}\right)$, such that $\boldsymbol{u}_{\mathrm{H}}$ is kinematically admissible, i.e. it verifies the kinematical relations and boundary conditions, $\boldsymbol{q}^{*}$ is statically admissible, i.e. it verifies the equilibrium equations in the strong form, and the pair $\left(\boldsymbol{u}_{\mathrm{H}}, \boldsymbol{q}^{*}\right)$ verifies the state equations (see the previous papers for a more precise definition). The error in constitutive relation is then computed as a function of this pair of solutions (see below). This approach was applied to a large class of linear and nonlinear problems (see the references in [3]). Note that, in linear solid mechanics, this approach to the error estimation problem is equivalent to the classical one [3], in the sense that the error indicators are the same.

The kinematically admissible solution is generally directly taken as the FE solution. For the computation of the statically admissible solution, a method was proposed [3], based on the resolution of local problems for each element of the original mesh. We propose here a new way to compute that field, following the lines described in the previous section, and therefore based on the resolution of local problems posed on stars $\Omega_{i}$.

Indeed, using the previous notations, it can be observed that the resolution of the local problems (2) yields a global stress field $\boldsymbol{q}^{*}=\boldsymbol{\sigma}\left(\boldsymbol{u}_{\mathrm{H}}\right)+\sum_{i} \boldsymbol{q}^{i}$ that verifies the following equations:

$$
\begin{cases}\operatorname{Div}_{\boldsymbol{x}}\left(\boldsymbol{q}^{*}\right)+\boldsymbol{f}=\mathbf{0} & \text { in } \Omega \\ \left(\boldsymbol{q}^{*}\right) \cdot \boldsymbol{n}=\boldsymbol{g} & \text { on } \Gamma^{\mathrm{N}}\end{cases}
$$

Hence, it is a solution to the initial problem, without the Dirichlet boundary condition on $\Gamma^{\mathrm{D}}$. It is therefore statically admissible, and it can be used, in pair with $\boldsymbol{u}_{\mathrm{H}}$, kinematically admissible, to compute the error in constitutive relation.

To simplify the formulations, and with no restriction on the domain of application, we consider a viscoelasticity problem described through internal variables (see for example [5]). The error in constitutive relation is taken as the dissipation error, and written

$$
\begin{aligned}
& 2 E_{\mathrm{d}}\left(\boldsymbol{u}_{\mathrm{H}}, \boldsymbol{q}^{*}\right)^{2}= \\
& \quad \int_{0}^{T} \int_{\Omega}\left(\dot{\boldsymbol{\epsilon}}_{\mathrm{H}}^{\mathrm{p}}-\mathcal{B}\left(\boldsymbol{q}^{*}\right)\right): \mathcal{B}^{-1}\left(\dot{\boldsymbol{\epsilon}}_{\mathrm{H}}^{\mathrm{p}}-\mathcal{B}\left(\boldsymbol{q}^{*}\right)\right) \mathrm{d} \Omega \mathrm{d} t
\end{aligned}
$$

where $\mathcal{B}$ is the constitutive tensor linking the stressrelated internal variables to the time derivatives of the internal variables related to the quasi-instantaneous strains $\dot{\boldsymbol{\epsilon}}^{\mathrm{p}}$, and $\dot{\boldsymbol{\epsilon}}_{\mathrm{H}}^{\mathrm{p}}=\dot{\boldsymbol{\epsilon}}_{\mathrm{H}}-\mathcal{K}^{-1}\left(\boldsymbol{q}^{*}\right)$ represents the internal variables representing the non-instantaneous strains of the FE solution $\boldsymbol{u}_{\mathrm{H}}$. In viscoelasticity, if $\mathcal{K}$ is the fourth order Hooke tensor relating the stress tensor and the linearized strain tensor, $\mathcal{B}$ is taken as $\mathcal{K}^{-1} / \tau$, where $\tau$ is some characteristic time. More generally, $\mathcal{B}$ has to derive from a convex pseudo-potential [25] for the following derivations to hold.

The dissipation error defined in that manner verifies the following two properties:

$$
\left\{\begin{array}{l}
E_{\mathrm{d}}\left(\boldsymbol{u}_{\mathrm{H}}, \boldsymbol{q}^{*}\right)^{2} \geq 0 \\
E_{\mathrm{d}}\left(\boldsymbol{u}_{\mathrm{H}}, \boldsymbol{q}^{*}\right)=0 \Leftrightarrow \boldsymbol{u}_{\mathrm{H}}=\mathcal{B}\left(\boldsymbol{q}^{*}\right)
\end{array}\right.
$$

and the second line also means that then, $\boldsymbol{u}_{\mathrm{H}}=\boldsymbol{u}_{\mathrm{ex}}$. Further, the dissipation error can be related to the exact solution through the following equation (see [3] for a proof):

$$
\begin{aligned}
\int_{0}^{T} 2 \Phi_{t}^{*}\left(\boldsymbol{\sigma}\left(\boldsymbol{u}_{\mathrm{ex}}\right)\right. & \left.-\frac{1}{2}\left(\boldsymbol{q}^{*}+\boldsymbol{\sigma}\left(\boldsymbol{u}_{\mathrm{H}}\right)\right)\right) \mathrm{d} t \\
& +E_{F}\left(\boldsymbol{\sigma}\left(\boldsymbol{u}_{\mathrm{ex}}\right)-\boldsymbol{q}^{*}\right)_{\mid t=T}=\frac{1}{2} E_{\mathrm{d}}\left(\boldsymbol{u}_{\mathrm{H}}, \boldsymbol{q}^{*}\right)^{2}
\end{aligned}
$$

where $\Phi^{*}(\boldsymbol{q})=\int_{\Omega} \boldsymbol{q}: \mathcal{B}\left(\boldsymbol{q}^{*}\right) \mathrm{d} \Omega$ is the integral over space of the conjugate dissipation pseudopotential of the stress field $\boldsymbol{q}$, and $E_{\mathrm{F}}(\cdot)$ is the free energy associated to a stress field. This equation is the equivalent, in the context of the dissipation error, of the inequality (3) in the context of the error in solution in linear solid mechanics, in the sense that it relates the error indicator to the exact solution.

\section{Application}

In this section, we present two applications that show the interest of the proposed method. Note that these error estimators could easily be used in adaptive strategies to refine the meshes locally, for example. 


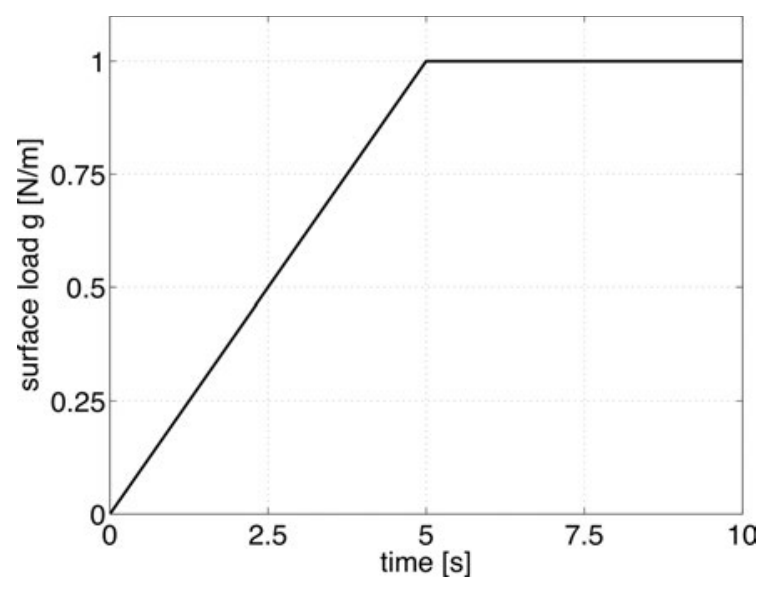

Fig. 1. Loading sequence.

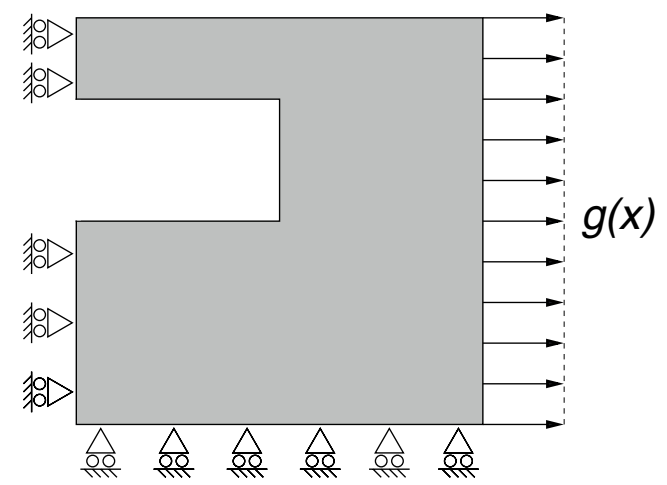

Fig. 2. Model of a quarter part of the perforated square plate.

\subsection{Perforated square plate}

The first application that we consider is that of a thin square plate with rectangular holes in 2D plane stress. That plate is loaded on the left and right sides by a ramplike unit normal traction (see Fig. 1). As the problem is symmetric both in the horizontal and vertical directions, the original problem is replaced by a quarter of the plate, with appropriate boundary conditions (see Fig. 2). The Young's modulus and the Poisson's ratio of the plate are taken as $E=1 \mathrm{~N} . \mathrm{m}^{-2}$ and $\nu=0.3$. The characteristic time is $\tau=0.5 \mathrm{~s}$. Due to the geometry, the solution of this problem is expected to show singularities in the corners of the interior hole, and the numerical errors to be concentrated there. Note that the same problem was already considered in several papers by various authors $[6,12,19,22,26]$, although, to the knowledge of the authors, it was not used in the context of viscoelastic problems. The time interval of study $[0, T]$ is discretized into 10 time steps, and we consider a backward Euler scheme for the time discretization.

Starting from a coarse mesh (80 nodes and 116 triangular elements), four embedded meshes are created, each time by splitting a triangle into four triangles. The three refined meshes have respectively 275, 1013 and 3881 nodes, and 464, 1856 and 7424 elements. In Figure 3, maps of the local contributions to the cumulative dissipation error $\int_{0}^{T} \int_{\Omega_{k}} E_{\mathrm{d}}^{2} \mathrm{~d} V \mathrm{~d} t / 2$ are plotted for each mesh. These

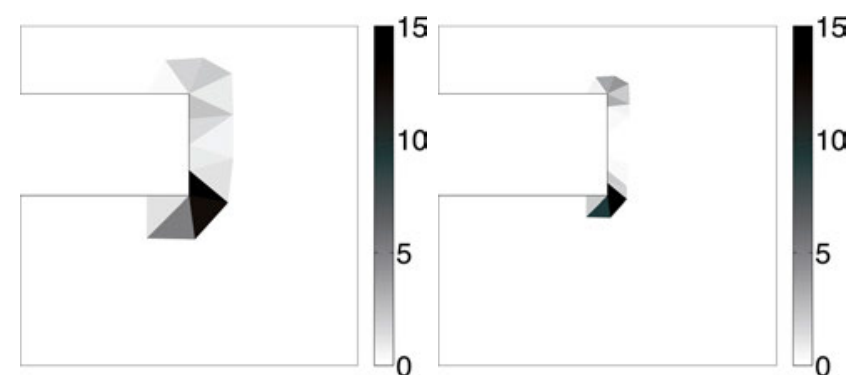

(a)

(b)

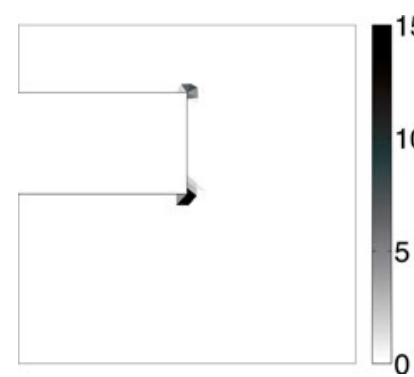

(c)

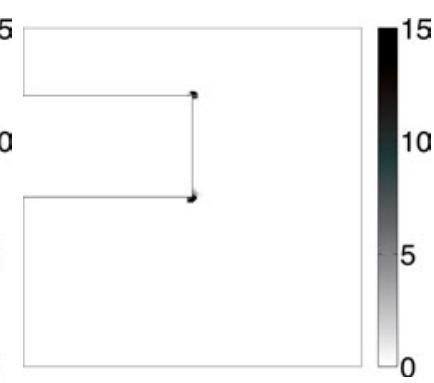

(d)
Fig. 3. Local cumulative dissipation error at the end of the loading sequence. The error is normalized by the area of the elements.

contributions are normalized by the area of each element $\Omega_{k}$ and considered at the end of the loading sequence $(t=10 \mathrm{~s})$. The singularity of the solution in the two corners of the rectangular plate can be clearly seen with the localization of the error in the very close vicinity of these corners.

In Figure 4, the evolution of the cumulative dissipation error $\int_{0}^{T} \int_{\Omega_{k}} E_{\mathrm{d}}^{2} \mathrm{~d} V \mathrm{~d} t / 2$ is shown as a function of time. A change of slope can be observed when the loading stabilizes to the constant value of 1 , but the dissipation error keeps increasing. Note that the behavior as a function of time can be modified by changing slightly the definition of the error with a weight function (see [5] for more details). Finally, the evolution of the error as a function of the number of elements (or, equivalently, the size of the elements) is plotted in Figure 5. Although the rate of decrease seems very small with respect to the classical $h^{\mathrm{p}}$ convergence, it should not be analyzed as a defect of the method. Indeed, these classical convergence results have been derived for the error in solution, and are only valid in the linear case. No such results are available in the nonlinear case, and for the dissipation error.

\subsection{Rectangular plate with a narrowing}

The second example we consider is that of thin rectangular plate with a narrowing in 2D plane stress (see Fig. 6). As in the previous example, the plate is loaded on the left and right sides by a ramp-like unit normal traction (Fig. 1). This time, we model the entire structure, with no use of the possible simplification arising from symmetry. The Young's modulus and the Poisson's ratio of the 


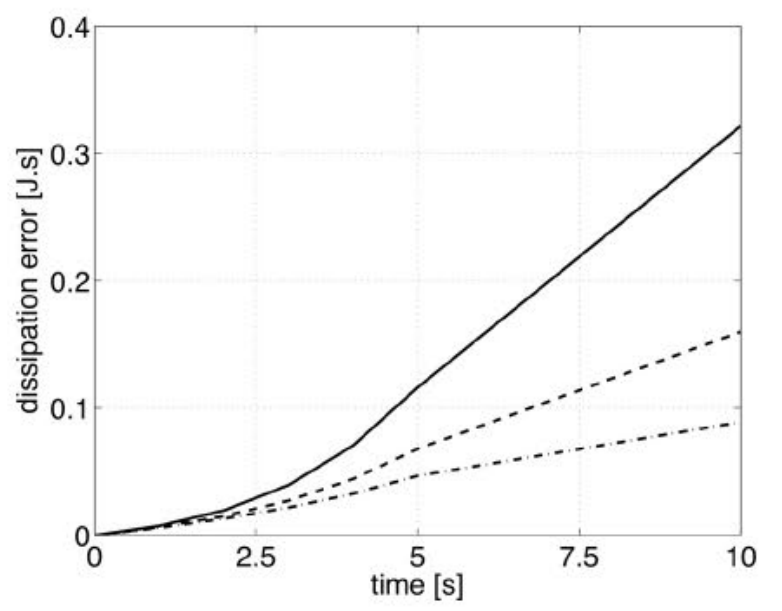

Fig. 4. Evolution of the cumulative dissipation error with the loading sequence.

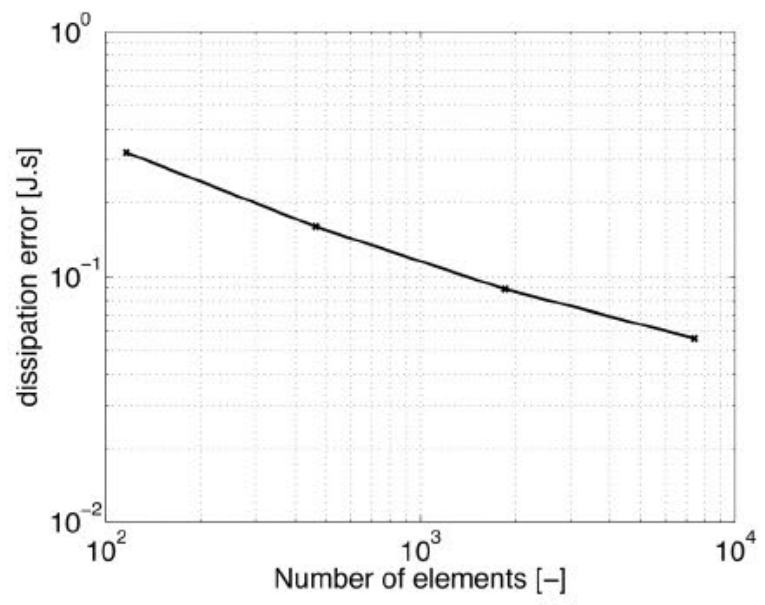

Fig. 5. Evolution of the cumulative dissipation error at the end of the loading sequence with the number of elements in the mesh.

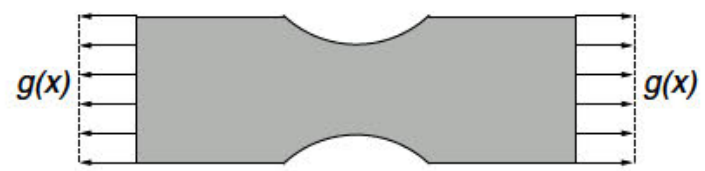

Fig. 6. Model of the rectangular plate with a narrowing.

plate are taken as $E=1 \mathrm{~N} . \mathrm{m}^{-2}$ and $\nu=0.3$. The characteristic time is $\tau=0.5 \mathrm{~s}$. The geometry should not yield singularities, and the errors are expected to be localized mainly in the sides of the plate, in the narrowing area, and close to the maximum value of the stresses.

Starting from a coarse mesh (117 nodes, 186 elements), two refined meshes are generated by splitting each triangle into three. The two additional meshes contain respectively 419 and 1581 nodes, and 744 and 2976 elements. Note that this refinement method means that the sequence of meshes does not converge towards the curved geometry of Figure 6. In particular, angular points remain along the curved boundaries at the location of the original elements in the coarse mesh. This is what is observed in the maps of the local contributions to the cumu-

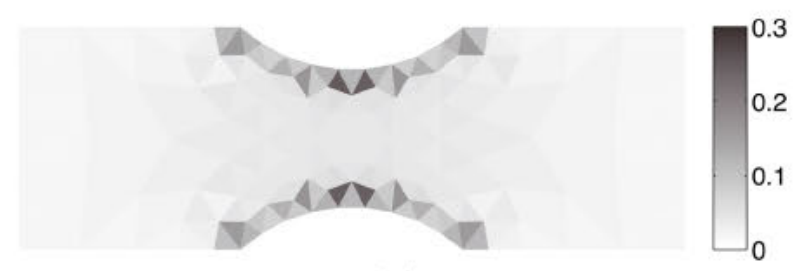

(a)

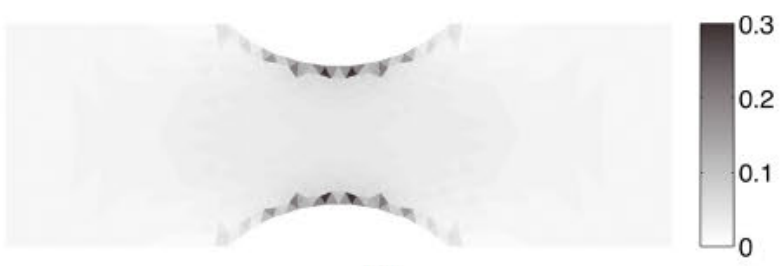

(b)

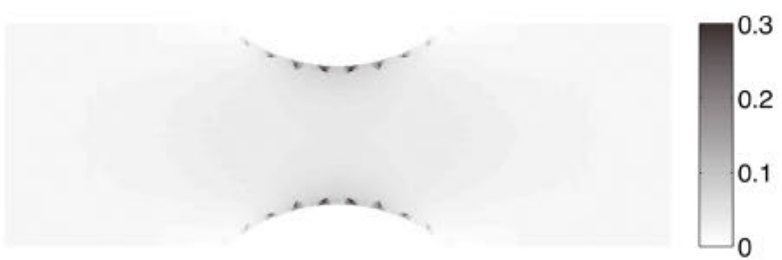

(c)

Fig. 7. Local cumulative dissipation error at the end of the loading sequence. The error is normalized by the size of the elements.

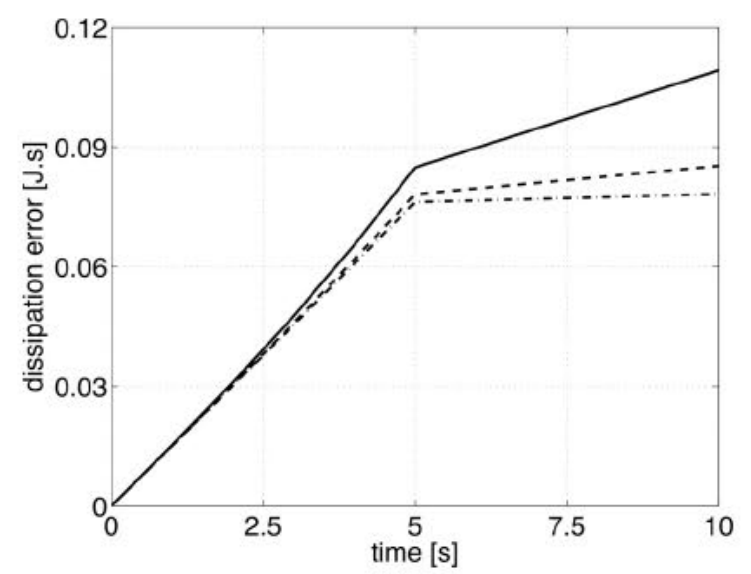

Fig. 8. Evolution of the cumulative dissipation error with the loading sequence.

lative dissipation error at the end of the loading sequence, where the error concentrates in these singularity points. It should be noted that, as expected, the error is much more spread out in that case than in the previous one. Indeed the values of the contributions throughout the domain are in the light gray range, while they were definitely white $(=0)$ in the case of the square plate.

In Figures 8 and 9 the evolution of the global dissipation error at the end of the loading sequence is plotted as a function of time and number of elements, respectively. The same remarks as in the previous case apply to this example. 


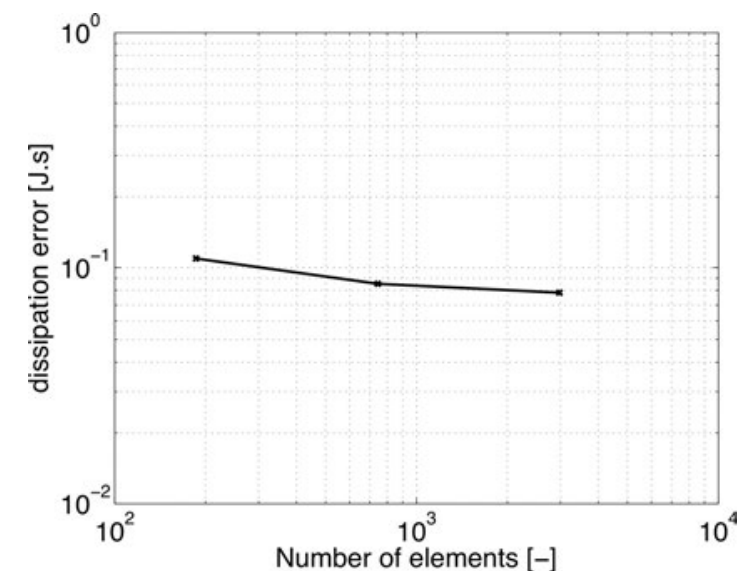

Fig. 9. Evolution of the cumulative dissipation error at the end of the loading sequence with the number of elements in the mesh.

\section{Conclusions}

The work that was presented in this paper can be seen in two equivalent ways: (1) as a way to extend the work described in [22] in the linear case to the nonlinear realm, or (2) as an alternative method to construct the equilibrated stress fields used in the dissipation error approach ([5] for example). Whatever the vision, it allows to compute efficiently an error estimator that can be used in adaptive strategies. Further, it can be generalized to the estimation of error in quantities of interest.

\section{References}

[1] M. Ainsworth, J.T. Oden, A posteriori error estimation in finite element analysis, Pure and Applied Mathematics, Wiley-Interscience, 2000

[2] N.E. Wiberg, P. Díez, Adaptive modeling and simulation, Comput. Methods Appl. Mech. Eng. 195 (2006) 205-480

[3] P. Ladevèze, J.-P. Pelle, Mastering calculations in linear and nonlinear mechanics, Mechanical Engineering, Springer, 2005

[4] P. Ladevèze, Upper error bound on calculated outputs of interest for linear and nonlinear structural problems, C. R. Mécanique 334 (2006) 399-407

[5] L. Chamoin, P. Ladevèze, Bounds on history-dependent or independent local quantities in viscoelasticity problems solved by approximate methods, Int. J. Numer. Meth. Eng. 71 (2007) 1387-1411

[6] P. Ladevèze, L. Chamoin, E. Florentin, A new nonintrusive technique for the construction of admissible stress fields in model verification, Comput. Methods Appl. Mech. Eng. 199 (2010) 766-777

[7] O.C. Zienkiewicz, J.Z. Zhu, A simple error estimator and adaptive procedure for practical engineering analysis, Int. J. Numer. Meth. Eng. 24 (1987) 337-357

[8] I. Babuška, W.C. Rheinboldt, Error estimates for adaptive finite element computations, SIAM J. Numer. Anal. 15 (1978) 736-755
[9] P. Ladevèze, D. Leguillon, Error estimate procedure in the finite element method and applications, SIAM J. Numer. Anal. 20 (1983) 485-509

[10] J.Z. Zhu, A posteriori error estimation - the relationship between different procedures, Comput. Methods Appl. Mech. Eng. 150 (1997) 411-422

[11] H.-W. Choi, M. Paraschivoiu, Adaptive computations of a posteriori finite element output bounds: a comparison of the "hybrid-flux" approach and the "flux-free" approach, Comput. Methods Appl. Mech. Eng. 193 (2004) 40014033

[12] N. Parés, P. Díez, A. Huerta, Subdomain-based flux-free a posteriori error estimators, Comput. Methods Appl. Mech. Eng. 195 (2006) 297-323

[13] C. Carstensen, S.A. Funken, Fully reliable localized error control in the FEM, SIAM J. Sci. Comput. 21 (1999-2000) 1465-1484

[14] L. Machiels, Y. Maday, A.T. Patera, A "flux-free" nodal Neumann subproblem approach to output bounds for partial differential equations, C.-R. Acad. Sci. Ser. I Math. 330 (2000) 249-254

[15] P. Morin, R.H. Nochetto, K.G. Siebert, Local problems on stars: a posteriori error estimators, convergence, and performance, Math. Comp. 72 (2003) 1067-1097

[16] S. Prudhomme, F. Nobile, L. Chamoin, J.T. Oden, Analysis of a subdomain-based error estimator for finite element approximations of elliptic problems, Numer. Methods Partial Differ. Eqs. 20 (2004) 165-192

[17] J.P. Moitinho de Almeida, E.A.W. Maunder, Recovery of equilibrium on star patches using a partition of unity technique, Int. J. Numer. Meth. Eng. 2008, submitted

[18] A.M. Sauer-Budge, J. Bonet, A. Huerta, J. Peraire, Computing bounds for linear functionals of exact weak solutions to Poisson's equation, SIAM J. Numer. Anal. 42 (2004) 1610-1630

[19] N. Parés, J. Bonet, A. Huerta, J. Peraire, The computation of bounds for linear-functional outputs of weak solutions to the two-dimensional elasticity equations, Comput. Methods Appl. Mech. Eng. 195 (2006) 406-429

[20] N. Parés, P. Díez, A. Huerta, Bounds of functional outputs for parabolic problems. Part I: Exact bounds of the discontinuous Galerkin time discretization, Comput. Methods Appl. Mech. Eng. 197 (2008) 1641-1660

[21] N. Parés, P. Díez, A. Huerta, Bounds of functional outputs for parabolic problems. Part II: Bounds of the exact solution, Comput. Methods Appl. Mech. Eng. 197 (2008) 1661-1679

[22] R. Cottereau, P. Díez, A. Huerta, Strict error bounds for linear solid mechanics problems using a subdomain-based flux-free method, Comp. Mech. 44 (2009) 533-547

[23] R. Becker, R. Rannacher, An optimal control approach to shape a posteriori error estimation in finite element methods, Acta Numerica 10 (2001) 1-102

[24] S. Prudhomme, J.T. Oden, On goal-oriented error estimation for elliptic problems: application to the control of pointwise errors, Comput. Methods Appl. Mech. Eng. 176 (1999) 313-331

[25] J.J. Moreau, Fonctionnelles convexes, Cours du Collège de France, 1966

[26] M. Paraschivoiu, J. Peraire, A.T. Patera, A posteriori finite element bounds for linear-functional outputs of elliptic partial differential equations, Comput. Methods Appl. Mech. Eng. 150 (1997) 23-50 